\title{
Provimento de Informações sobre Transporte Público Urbano para Empresas Concessionárias: Simulação e Avaliação Apoiada por um Sistema Multi-agentes
}

\author{
Thiago Lopes Trugillo Silveira e Marcia Pasin ${ }^{1}$
}

${ }^{1}$ Centro de Tecnologia - Universidade Federal de Santa Maria (UFSM)

Av. Roraima 1.000 - Cidade Universitária - Santa Maria - RS - Brasil

\{thiago, marcia\}einf.ufsm.br

\begin{abstract}
Monitoring and planning are interesting activities to help transit operator and to provide quality in public transportation. In fact, some computational tools and simulators play this role and assist humans in tasks and improving transportation. However, existing solutions are typically proprietary and, therefore, have prohibitive cost to be adopted by small and medium size cities. In addition many of them are not easy to be operated by non-specialists. In this paper, we describe the implementation and evaluation of a simulation using the support of a multi-agent system to represent dynamic components (flow, roads, vehicles, pedestrians, bus stops, etc.) in the urban transport. Our simulation can be easily operated by non-specialists and was experimentally evaluated using as scenario a medium city, considering the variations of two given journeys.
\end{abstract}

Resumo. O monitoramento e planejamento por empresas concessionárias são elementos chave na tarefa de provisão de transporte público com qualidade. De fato, algumas ferramentas computacionais e simuladores desempenham este papel e auxiliam humanos nas tarefas de melhoramento e gestão de transporte. No entanto, soluções existentes são tipicamente proprietárias, com custo proibitivo para muitas cidades, ou então não são fáceis de usar por não-especialistas. Este trabalho apresenta a implementação e avaliação de uma simulação apoiada por um sistema multi-agentes para modelar os componentes de transporte urbano coletivo (como fluxo, vias, veículos, pedestres, paradas de ônibus). A ferramenta pode ser facilmente operada por não-especialistas e foi avaliada experimentalmente usando como cenário uma cidade de médio porte, considerando variações de fluxo do período de diferentes jornadas completas.

\section{Introdução}

A crescente complexidade da gestão do transporte nas cidades tem exigido, por órgãos gestores (municípios, empresas concessionárias) e usuários deste serviço, a convenção de novas regras e a implantação de melhorias, visando garantir operação mais eficiente. Com o objetivo de automatizar processos e prover suporte computacional adequado, Sistemas Inteligentes de Transporte (ITS ou Intelligent Transport Systems) estão sendo cada vez mais implementados e desenvolvidos. Seu uso possibilita previsão, análise e diagnóstico para problemas no processo de tráfego de veículos e circulação de pedestres.

Um aspecto importante no processo de transporte público é o desenvolvimento de ferramentas de monitoramento e de simulação que permitam aos órgãos gestores acesso 
fácil e preciso a informações, motivado pela grande importância no melhoramento do serviço (mais ônibus, menos carros). Estas ferramentas devem fornecer informações sobre componentes desta problemática (como fluxo, vias, veículos, pedestres, paradas de ônibus) que viabilizem planejamento operacional e gestão (através de visualização de comboios, gargalos devido a acidentes, saídas de espetáculos, entre outros, e geração de relatórios estatísticos) e, finalmente, melhoria da Qualidade de Serviço $(Q o S)$ para o usuário final.

É importante fornecer, a órgãos gestores, informações para facilitar planejamento tais como aquelas que medem os efeitos do nível de serviço das vias, e o número de veículos atrasados. Por exemplo, dado um certo nível de serviço, em um certo período do dia, qual é o número de veículos a serem colocados em uma linha particular para obter determinada $Q o S$ ? No entanto, um obstáculo no caso de transporte público urbano é a ausência de ferramentas de fácil operação por não-especialistas, de baixo custo de aquisição para que possam ser adotadas por cidades de pequeno e médio porte, e que permitam o planejamento, provisionamento de informação e monitoramento em tempo real, além da execução de simulações. Existe carência de investimento nessas ferramentas e o resultado se reflete na dificuldade de gestão, que impacta fortemente na $Q o S$. Considerado um elemento chave neste processo, ferramentas de simulação de software permitem a avaliação de situações, possibilitando a observação de diferentes cenários e antecipação de detecção de problemas, antes mesmo da implementação de soluções na prática.

Desta forma, este artigo apresenta o desenvolvimento e avaliação de uma simulação gráfica para fornecer informações aos órgãos gestores (notadamente empresas concessionárias) do sistema de transporte público urbano apoiada por um sistema de multiagentes (MAS ou Multi-Agent System). O conceito de agentes de software permite a modelagem de cada componente desta problemática de forma independente e dinâmica, associando ações específicas para atribuir maior realismo ao software resultante.

Mais precisamente, a simulação aqui apresentada destaca as seguintes características: $(i)$ o uso de software aberto e de livre distribuição, enfatizando baixo custo e liberdade de distribuição e modificação, inclusive por órgãos gestores de cidades de pequeno e médio porte $(i i)$ usabilidade porque a ferramenta precisa de ser fácil de ser usada e compreendida por não-especialistas, (iii) possibilidade de observação de comportamento dinâmico de fluxos no processo de transporte público, suportado por um MAS, $(i v)$ simulação de fluxos levando em conta diferentes etapas do dia (para demostrar movimentação na hora do rush e horas de ociosidade), $(v)$ geração de relatórios e coleta de dados estatísticos. A simulação foi avaliada experimentalmente no contexto de uma cidade de pequeno porte, considerando as variações do período de uma jornada completa.

Em geral, a simulação aqui proposta visa servir como uma ferramenta para permitir melhor compreensão e agilizar o processo de entendimento de problemas no sistema de transporte nas cidades de médio e pequeno porte. Além disso, essas informações podem ser utilizadas por empresas licenciadas para antecipar demandas e tomar ações pró-ativas para melhorar a $Q O S$ de transporte e gestão de frotas.

O restante deste artigo está organizado da seguinte forma. Trabalhos relacionados são apresentados na seção 2. A Seção 3 apresenta o detalhamento da simulação para transporte público urbano. Seção 4 aborda aspectos de implementação. Seção 5 descreve 
a avaliação experimental da simulação usando como cenário um conjunto de linhas que servem uma cidade de porte médio. Finalmente, a Seção 6 apresenta conclusões finais e trabalhos futuros.

\section{Trabalhos relacionados}

O provimento de transporte público eficiente é um grande desafio para o desenvolvimento de cidades inteligentes. Visando mobilidade sustentável, as Tecnologias de Informação e Comunicação (TIC) têm sido aplicadas, com o resultado primário dos ITS. Neste sentido, diversos aspectos têm sido ser explorados, tais como a construção de $(i)$ simuladores muitas vezes apoiados por MAS, $(i i)$ ferramentas que oferecem informação online sobre tráfego e trânsito em geral (Google Traffic, Google Transit, Waze e outros aplicativos para GPS), e (iii) ferramentas e sistemas para entendimento e gerenciamento de transporte público especificamente voltadas para empresas.

No contexto de simuladores, destacam-se soluções proprietárias (VisSim, http: //www.vissim.com, entre outras) que apresentam o inconveniente do alto custo de implantação e manutenção, e soluções acadêmicas (SUMO [Behrisch et al 2011], Itsumo [Castro da Silva 2004]), tipicamente suportados por MAS. Simuladores não são feitos para serem usados por não-especialistas e, tipicamente, não identificam componentes da problemática de transportes de forma individualizada (por exemplo, nome de uma determinada linha de ônibus). Há a necessidade de uma camada de software para fazer a interface-simulador usuário e, este presente trabalho contribui neste sentido. A implementação aqui apresentada foi conduzida sobre o simulador o Siafu por sua facilidade de desenvolvimento e pela disponibilidade deste software que é de livre distribuição.

Existe também uma coleção de ferramentas, disponíveis gratuitamente, que possibilitam observar movimentação de veículos em geral. Um exemplo é o GoogleTraffic (http://maps.google.com.br/) que apresenta o comportamento do tráfego nas vias urbanas com o suporte de crowd sourcing. Usuários de transporte quando utilizam serviços Google em seus dispositivos móveis com GPS, implicitamente enviam informações (posição atual e velocidade) para o centro de operações do Google. Com base nos dados recebidos e histórico, a ferramenta fornece uma estimativa do tráfego que é exibido usando cores diferentes em um mapa do Google Maps, através de interface web. Outro exemplo é a ferramenta colaborativa Waze (http: / / www . waze.com) também baseada em GPS. O Waze permite que usuários colaborem na inserção de informações relevantes para o andamento do fluxo de transporte e trânsito.

Ferramentas e sistemas para entendimento e gerenciamento de transporte público também têm sido desenvolvidas nas empresas, como o sistema Somar [Ladeira el al. 2009] da Carris de Porto Alegre em desenvolvimento a partir de 1997, o sistema alemão INITRANS [Staib e Gerland 1999], que começou de forma acadêmica de depois tornou-se um produto, e tantas outras ferramentas proprietárias (Mentor Streets Transit (http://www.mentoreng.com/), FLASHNET (http: //www.swarco.com), Fleet Focus Transit (http://www.assetworks.com/ fleet-management-software/), IVU.FLEET (http://www.ivu.com/)). Ferramentas proprietárias apresentam características muito úteis (entre elas destaca-se a localização automática de veículos, e o envio de mensagens para motoristas), inclusive algumas podem ser usadas tanto em modo standalone, para simulação, por exemplo, ou 
em modo tempo real. Contudo, requerem investimento substancial na implantação e na manutenção da sua operacionalidade, o que pode ser inviável para a realidade de muitas cidades brasileiras.

Finalmente, destacam-se trabalhos acadêmicos como ênfase em técnicas de inteligência artificial. [Molina 2005] apresenta um sistema de recomendação para diagnóstico, previsão e planejamento de transporte público. Outras simulações, baseadas em MAS, são descritas em [Meignan et al 2007] e [Gruer et al 2001]. Entretanto, o problema destas ferramentas é que elas permitem apenas um número limitado de funções e relatórios (por exemplo tempo de espera do passageiro, variação de demanda de passageiros), e não são voltadas para o usuário leigo. Detecção de comboios é um assunto não abordado.

De forma geral, existe uma carência de ferramentas, aplicações e sistemas de informação que permitam o entendimento, o monitoramento e o planejamento, por parte das instituições, de serviços de transporte público urbano além do provimento de informação para o usuário deste tipo de transporte. Estas ferramentas e sistemas, se implementados de forma adequada (desempenho eficiente, resultados corretos e em tempo hábil, disponibilidade, e facilidade de uso), podem oferecer suporte interessante para observação, controle, previsão e simulação de diferentes cenários.

\section{Solução proposta: provimento de informações para operadoras de trânsito}

Visando oferecer uma simulação amigável e com baixo custo de implantação, neste trabalho foi desenvolvida uma implementação com o auxílio de uma biblioteca de simulação livre (Siafu) [Martin e Nurmi 2006], baseada em MAS. Esta simulação foi avaliada usando como cenário um conjunto de linhas que servem a cidade de Santa Maria - RS. Esta solução é descrita a seguir.

\subsection{Arquitetura}

A arquitetura da simulação é estruturada em três camadas: entrada de dados, processamento e visualização. O módulo de entrada de dados é alimentado com informações estáticas como posição geográfica paradas de ônibus (valores para latitude e longitude) e descrição de paradas que compõem as rotas que são previamente gravadas em armazenamento não-volátil. Essas informações são alimentadas por uma infraestrutura de suporte que permite a coleta das informações, através de sensores, sobre a frota de veículos que oferece o transporte público urbano.

O módulo de entrada de dados suporta o módulo de processamento. O módulo de processamento é apoiado pelo Siafu, e envolve cálculo de valores usados no módulo de visualização. O módulo de visualização integra geração de relatórios (ver item 3.2) e provimento de informações para a interface gráfica. A Figura 1 apresenta um screenshot da implementação atual da interface gráfica, com duas áreas principais: mapa com veículos em descolamento e área de visualização de informações sobre um determinado veículo (selecionado através de um menu). Usando a interface gráfica, um usuário comum pode realizar simulações e previsões para obter informações com a finalidade de facilitar a gestão do sistema de transporte público urbano. O usuário tem as opções de parar uma simulação e de alterar a velocidade da simulação.

De forma mais específica, com o auxílio da interface gráfica, o usuário pode observar as seguintes informações: $(i)$ informações estáticas sobre carros (como identificação, 


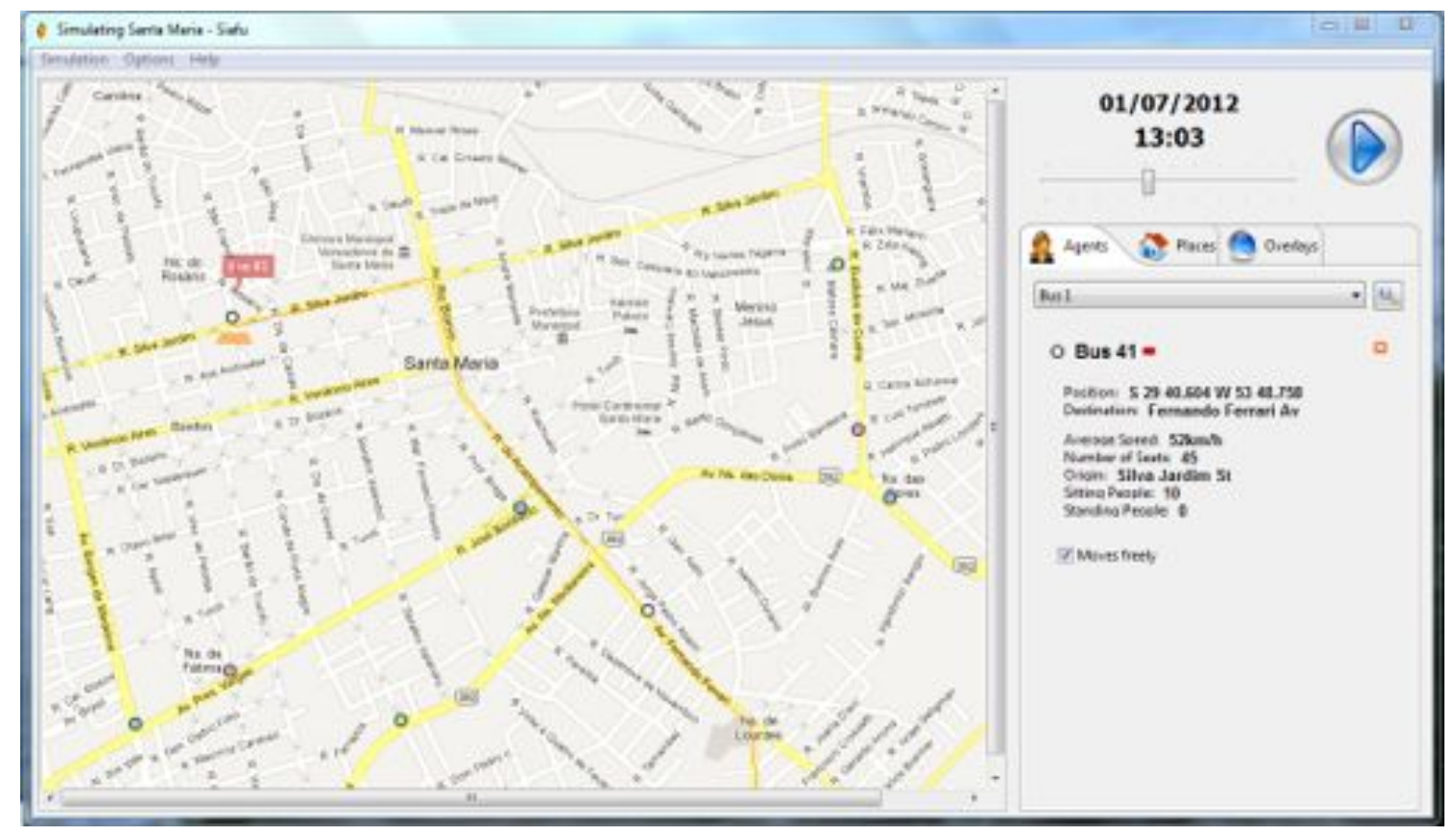

Figura 1. Screenshot da implementação atual da interface gráfica de simulação

através do número da placa do veículo, por exemplo) e linhas associadas (nome da linha, origem e destino), (ii) informação dinâmica sobre a localização do ônibus, (iii) a velocidade média do veículo, $(i v)$ consumo médio de combustível, $(v)$ número atual de passageiros no ônibus (passageiros sentados e em pé) e $(v i)$ informações avançadas, como detecção de comboios.

Ainda falta projetar e implementar facilidades de edição de mapas. Uma vez finalizada a implementação da ferramenta, as simulações podem ser feitas para o tráfego através da $(i)$ adição ou remoção de uma parada de ônibus em uma linha, $(i i)$ adição ou remoção de um ônibus em uma linha, ou ainda (iii) parada um veículo para simular o acontecimento de um acidente.

\subsection{Geração de relatórios}

Outro recurso implementado pela ferramenta, além da simulação visual, é a geração de um conjunto de relatórios ao final de cada simulação. Os relatórios descrevem diferentes listagens que podem ser usadas como medida de $Q o S$ e auxiliam no processo de tomada de decisão. O relatórios descrevem $(i)$ os carros mais atrasados, $(i i)$ as linhas mais sobrecarregadas e (iii) comboios detectados. Estes relatórios são gerados no final da jornada de simulação. Na implementação atual, a visualização é feita através de um arquivo HTML.

Os carros mais atrasados são detectados através de um cálculo simples da diferença entre o tempo esperado para o cumprimento de um percurso e do tempo que o ônibus levou para cumprir o percurso em uma simulação. Atualmente, o relatório apresenta uma listagem com o ID, tempo de saída da garagem, tempo aguardado para percorrer o percurso, tempo que o ônibus levou para percorrer o percurso e identificação do percurso/linha. Este relatório será alterado oportunamente para apresentar não apenas informações da simulação mas também dados estatísticos como informações como um indicador de pontualidade para cada linha. 
O relatório das linhas mais sobrecargas lista uma série de linhas mais congestionadas. O cálculo é feito medindo o fluxo de usuários que entram e saem em cada veículo. Valores altos para este índice em uma determinada linha demostram que esta linha está no elenco das mais congestionadas.

Outra funcionalidade implementada pela ferramenta aqui proposta é a detecção de comboios. Um comboio é formado por um grupo de carros (dois ou mais) que viajam juntos, de acordo com um determinado destino. O objetivo de um comboio é aumentar o fluxo de embarque e desembarque de ônibus em uma parada regular para evitar o congestionamento em corredores de ônibus dedicados. Tipicamente, um congestionamento nos pontos de interesse é causado pelo tempo necessário para o embarque e desembarque de passageiros num carro. A eficiência do comboio depende do tamanho médio do comboio (TMC) e também no fluxo de usuários do serviço. A implementação deste serviço é detalhada na no item 4.3 deste artigo.

Finalmente, quando todo o sistema se tornar operacional, sensores instalados na infraestrutura de transporte (em ônibus, paradas) coletariam dados sobre o posicionamento de ônibus, a velocidade, o volume de combustível no tanque e enviariam, em tempo real, informações para atualizar o banco de dados. Estas informações serão obtidas a cada intervalo fixo de tempo. Como alternativa, a mesma informação pode ser usada para fornecer informações para um serviço de informação avançada ao usuário (ATIS ou Advanced Traveler Information System), com auxílio de monitores e dispositivos móveis.

\section{Implementação}

A ferramenta de simulação, elemento chave para o entendimento e planejamento do processo de transporte público urbano, foi implementada tendo como base mapas reais e o uso de informações previamente armazenadas em arquivos em formato CSV chamados Buses.csv, Routes.csv e Schedule.csv, respectivamente. Conforme já comentado neste texto, o simulador Siafu foi usado como suporte.

\subsection{Siafu}

Siafu (http://siafusimulator.sourceforge. net) é um conjunto de ferramentas de simulação, desenvolvido no contexto de SMA. Para programar usando Siafu, três classes básicas devem ser estendidas: BaseAgentModel, BaseWorldModel e BaseContextModel. Estas classes tratam respectivamente do comportamento dos agentes (agentes podem executar diferentes ações dentro da simulação, ônibus e paradas, neste cenário), lugares (há lugares específicos onde os ônibus podem circular, ou seja, as linhas) e sobreposições (que são as regiões do mapa que se destinam a causar alterações na simulação). As sub-classes (classes que estendem as três classes de base acima mencionadas) precisam substituir os métodos de criação $x$ (onde $x$ representa agentes, lugares ou sobreposições) e iterações entre eles. O primeiro é responsável por inicializar cada um dos modelos (agente e contexto em questão) e as últimas atualizações em cada um deles a cada iteração da simulação.

De fato, a simulação em questão destina-se a mostrar a exploração de linhas ônibus de uma determinada micro-região (ou seja, Santa Maria). Então, foi implementada uma simulação onde ônibus viajam nesta micro-região como uma extensão do agente de classe $(\mathrm{Siafu} *$ ) que guardam informações tais como: origem, destino, número de lotação, capacidade de passageiros, velocidade. Uma simulação de um cenário com paradas 
de ônibus e ingresso/abandono de passageiros é feito atribuindo o comando parada de onnibus para certos lugares Siafu. De fato, existem dois tipos de locais onde os ônibus exercem atividades: garagem da operadora de serviço e paradas de ônibus.

Assim, foi implementado um sub-conjunto de serviço de linhas de ônibus através da criação de vários agentes a partir de um ponto de partida (garagem) que se desloca para o mesmo lugar final (garagem), com execução de serviço em paradas ao longo do caminho. Em cada lugar de conhecimento pelo Siafu, cada parada de ônibus tem informações sobre quantas pessoas estão à espera de um ônibus. Esta quantidade de pessoas muda ao longo do experimento e também muda quando um carro atinge uma parada. Para simplificar a implementação, na simulação atual, o ônibus tem velocidade constante, ou aceleração não está presente. Eles permanecem na mesma velocidade, sempre indo de um lugar a outro, utilizando como ponto de partida uma garagem e terminando em um ponto final, ou de um ponto de ônibus. Quando um ônibus encontra uma parada, espera até as pessoas que estão aguardando entrarem no ônibus, como ocorre na realidade. Esta parte da simulação é feita através de uma chamada em espera (neste caso, a velocidade do ônibus em questão é zero), multiplicando o número de pessoas que vão entrar ou sair do ônibus por uma constante (tempo médio que leva cada pessoa a subir ou deixar o ônibus).

\subsection{Overlays}

O Siafu comporta o uso de diferentes overlays (isto é mapas onde se pode manipular a frequência de objetos atuantes), então este esquema foi usado para modelar a quantidade de passageiros que embarcam e desembarcam em cada ônibus, de acordo com o período de duração da jornada. A Figura 2 mostra o esquema de overlays usado na simulação.

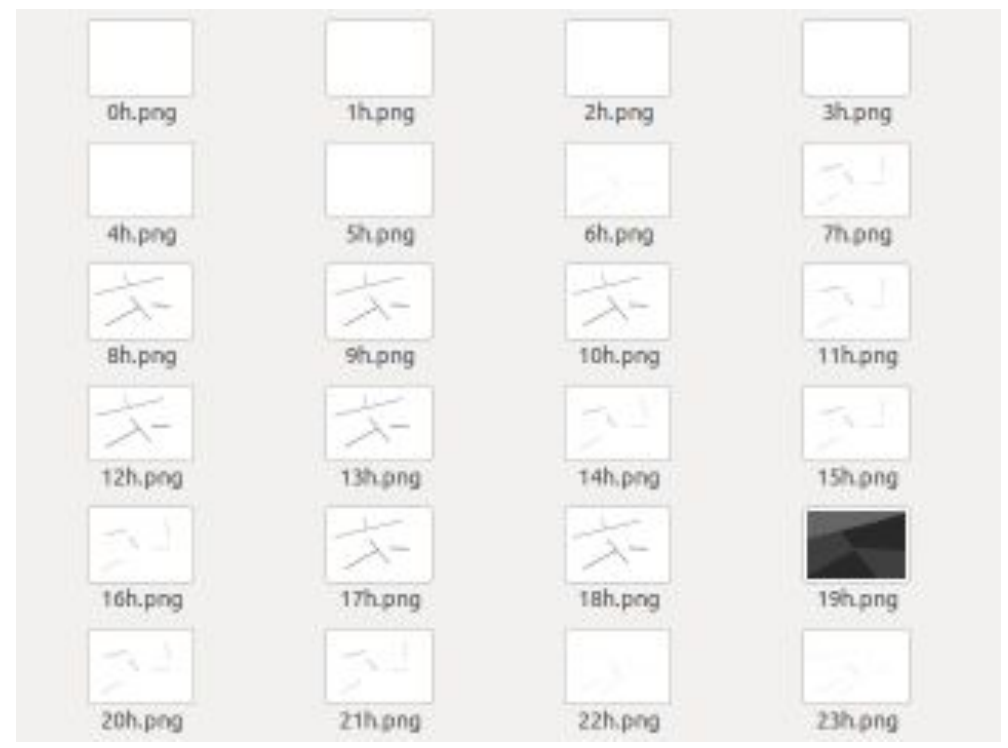

Figura 2. Diferentes overlays usados nas simulações de uma jornada completa

Em uma jornada típica, cedo da manhã, as pessoas deixam suas casas rumo ao trabalho ou à universidade, há grande concentração de pessoas embarcando e desembarcando nos ônibus. Durante a metade da manhã, o fluxo de passageiros diminui e volta a aumentar no período do meio-dia. Decresce no meio da tarde e volta a crescer no final da tarde quando as pessoas deixam o local de trabalho ou de estudo. 


\subsection{Detecção de comboios}

Detecção de comboios é um serviço relativamente fácil de ser implementado pelo Siafu. Siafu fornece um método chamado isNear que devolve verdadeiro se um agente está próximo ao outro, que foi usada para aplicar a detecção de comboio. Como o método isNear é aplicado a todos os carros na simulação, um comboio será eventualmente detectado.

O Algoritmo 1, contribuição deste trabalho, esboça a descoberta de comboio entre dois ou mais ônibus usada na simulação. Nas linhas 1 até 5 é feita a seleção dos agentes (ônibus) que estão sendo exibidos na interface, ou seja, os ônibus visíveis. Após, nas linhas 6 e 7, faz-se a comparação entre todos os ônibus visíveis. Durante a comparação, na linha 8, é testada a proximidade (de acordo com um FACTOR de proximidade prédefinido) entre dois agentes distintos $b u s_{i}$ e $b u s_{j}$ que pertencem a mesma linha de ônibus: se o teste é positivo, as imagens destes agentes são alteradas para uma imagem que sinaliza um comboio para facilitar a visualização. Finalmente, na linha 11, as imagens originais são restauradas caso a distância entre dois agentes não esteja dentro da pré-estabelecida.

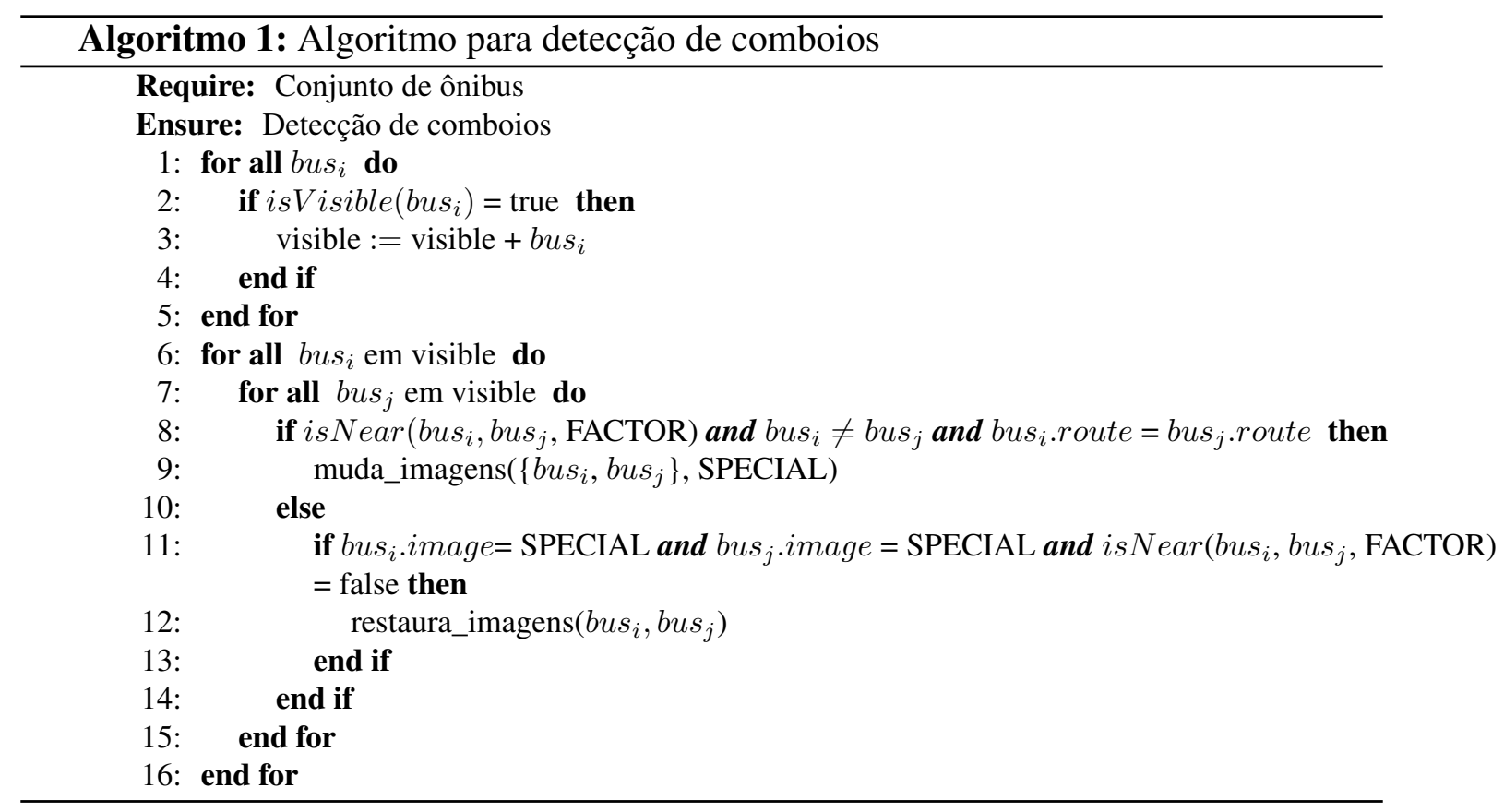

\section{Avaliação experimental}

Para avaliar a ferramenta aqui proposta e implementada, foi conduzido uma análise experimental usando como cenário um conjunto de linhas do transporte público urbano da cidade de Santa Maria - RS. Diferentes fluxos foram submetidos, fixando o número de paradas e ônibus e alterando a quantidade de passageiros que embarca ou desembarca em um ônibus durante uma jornada. De acordo com os experimentos efetuados, foi observado a $Q o S$ em termos de passageiros sentados e em pé, frequiência de lotação máxima de ônibus e incidência de linhas com mais atrasos. O cenário usado para o experimento é composto por dois fluxos distintos um deles composto por $(i)$ um conjunto de 6 linhas, 15 paradas e 188 ônibus e outro composto por (ii) 70 paradas, 12 linhas, 700 ônibus. A partir deste ponto do texto, o primeiro fluxo será chamado de fluxo reduzido e o segundo fluxo será chamado de fluxo ampliado. 


\subsection{Fluxo reduzido}

Uma simulação com uma jornada de 24 horas (0h-23h) e outra para o período da manhã (8h30-12h) foram realizadas com o fluxo reduzido. A Tabela 1 mostra informações sobre o número de viajantes em pé e sentados em relação à frequência de serviço de ônibus para intervalos de partida da garagem de ônibus de 30/60/90 minutos, respectivamente, obtida com dados da simulação.

\begin{tabular}{|l||l||l||l|}
\hline Passageiros & $30 \mathrm{~min}$ & $60 \mathrm{~min}$ & $90 \mathrm{~min}$ \\
\hline \hline Sentados & 28,14 & 42,28 & 42,95 \\
\hline Em pé & 0,22 & 8,43 & 26,90 \\
\hline
\end{tabular}

Tabela 1. Listagem com quantidade de passageiros sentados e em pé em função de diferentes frequências de serviço para uma determinada linha no serviço da manha para o fluxo reduzido

Para obter esses resultados foram executadas 10 simulações levando em conta o serviço da manhã. Pode ser observado que para o cenário de entrada, para fornecer o melhor nível de $Q o S$ para os viajantes (isto é, todos ou quase todos sentados), um serviço de transporte tem de ser iniciado a cada $1 / 2$ hora (apenas 0,22 passageiros em pé, em média, nesta frequência).

A Tabela 2 apresenta uma listagem como o comboio detectado para o fluxo reduzido na jornada de 24 horas. Apenas um comboio foi detectado.

\begin{tabular}{|l||l||l||l|}
\hline Hora do dia & bus $_{i}$ & bus $_{j}$ & Nome da linha \\
\hline \hline $12: 43$ & Bus 176 & Bus 175 & Euclides da Cunha St - N. Sra Medianeira Av \\
\hline
\end{tabular}

Tabela 2. Comboios detectados em uma jornada de 24 horas

A Tabela 3 apresenta uma listagem com linhas mais atrasadas para uma determinada jornada para o fluxo reduzido. A tabela está ordenada de acordo com o campo Indicador de atraso que identifica que a linha mais atrasada é Euclides da Cunha $S t-N$. Sra Medianeira Av e que a linha mais pontual é Presidente Vargas Av - N. Sra Dores Av.

\begin{tabular}{|l||l||l||l|}
\hline Nome da linha & $\begin{array}{l}\text { Atraso total } \\
\text { (em minutos) }\end{array}$ & $\begin{array}{l}\text { Qtde total } \\
\text { de ônibus }\end{array}$ & $\begin{array}{l}\text { Indicador } \\
\text { de atraso }\end{array}$ \\
\hline \hline Euclides da Cunha St - N. Sra Medianeira Av & 10 & 9 & 1,11 \\
Silva Jardim St - Fernando Ferrari Av & 4 & 2 & 2,00 \\
N. Sra Dores Av - Presidente Vargas Av & 28 & 8 & 3,50 \\
Fernando Ferrari Av - Silva Jardim St & 23 & 6 & 3,83 \\
Presidente Vargas Av - N. Sra Dores Av & 31 & 6 & 5,16 \\
Presidente Vargas Av - Euclides da Cunha St & 131 & 22 & 5,94 \\
\hline
\end{tabular}

Tabela 3. Listagem com linhas mais atrasadas para fluxo reduzido em uma jornada de 24 horas

\subsection{Fluxo ampliado}

Para o fluxo ampliado, foi realizada uma simulação de uma jornada de 24 horas. Foram avaliadas as seguintes métricas: quantidade de passageiros sentados versus frequência de ônibus, quantidade de passageiros em pé versus frequência de ônibus, e tempo médio de 
espera. A Figura 3 descreve o fluxo ampliado. Pode ser observado que há uma variação na quantidade ônibus em percurso, notadamente após as 6 horas da manhã. A quantidade de ônibus por hora usada na simulação foi gerada com o auxílio do recurso do Siafu de overlay usando os mapas descritos na Figura 2.

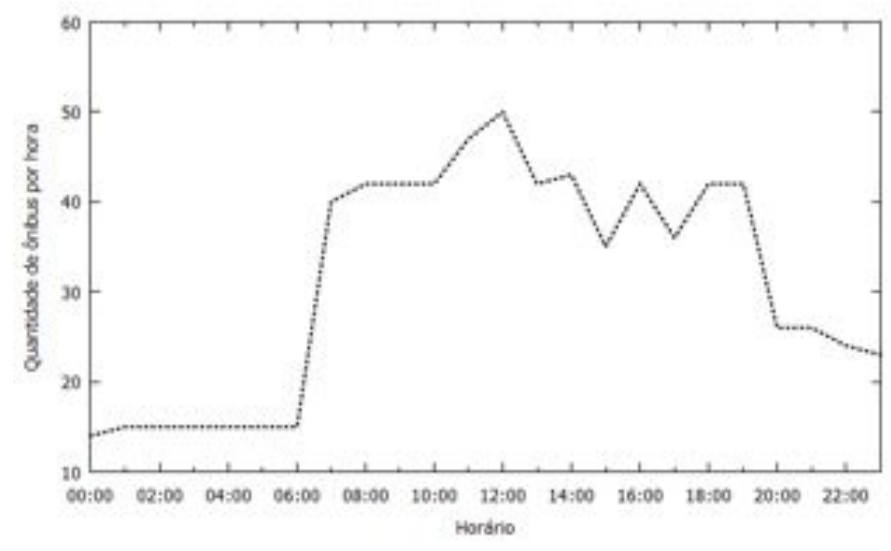

Figura 3. Descrição do fluxo ampliado na jornada de 24 horas

Levando em conta o fluxo ampliado, descrito através do gráfico da Figura 3, as Figuras 4 e 5 apresentam, respectivamente, os gráficos com o número de passageiros sentados e em pé, dada uma certa frequência de ônibus (15/20/30 minutos). Pode ser observado, que o aumento da frequência do ônibus influencia na quantidade de passageiros sentados e em pé, exceto em horários entre a meia-noite e as 5 horas da manhã.

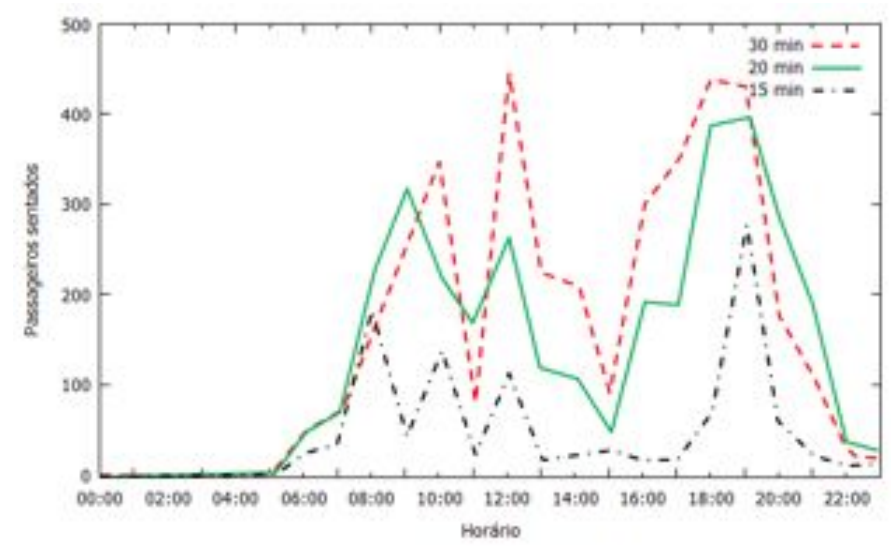

Figura 4. Passageiros sentados para o fluxo ampliado em uma jornada de 24 horas para intervalos de 15/20/30 minutos (média de todas as linhas)

O gráfico da Figura 6 descreve o tempo médio de espera para uma simulação na jornada de 24 horas em fluxo ampliado. Para o fluxo avaliado, o serviço deverá contar com mais de 50 ônibus em circulação para que o tempo de espera de um usuário em uma parada seja inferior a 10 minutos. Finalmente, o gráfico da Figura 7 descreve a quantidade de passageiros por ônibus (em pé ou sentados) na jornada de 24 horas em fluxo ampliado (média de todas as linhas). Os picos ocorrem no final das jornadas. 


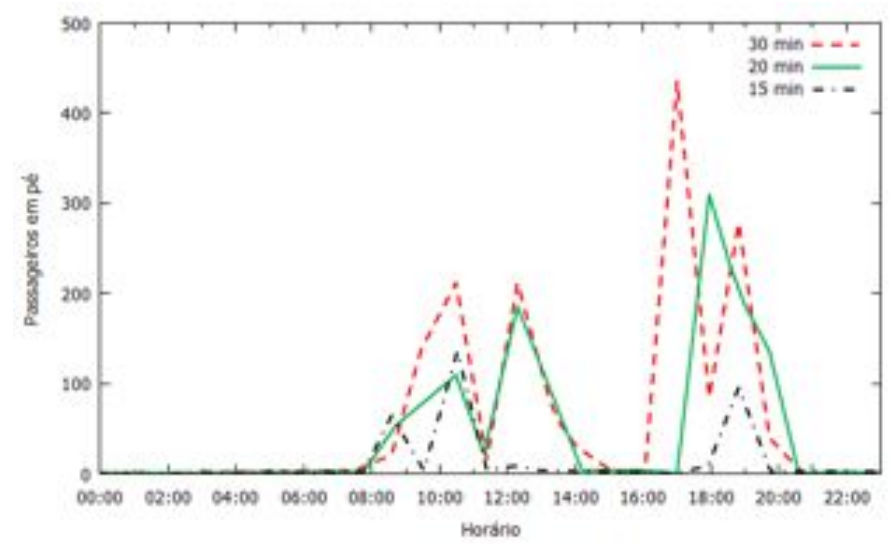

Figura 5. Quantidade de passageiros em pé no fluxo ampliado por jornada de 24 horas (média de todas as linhas)

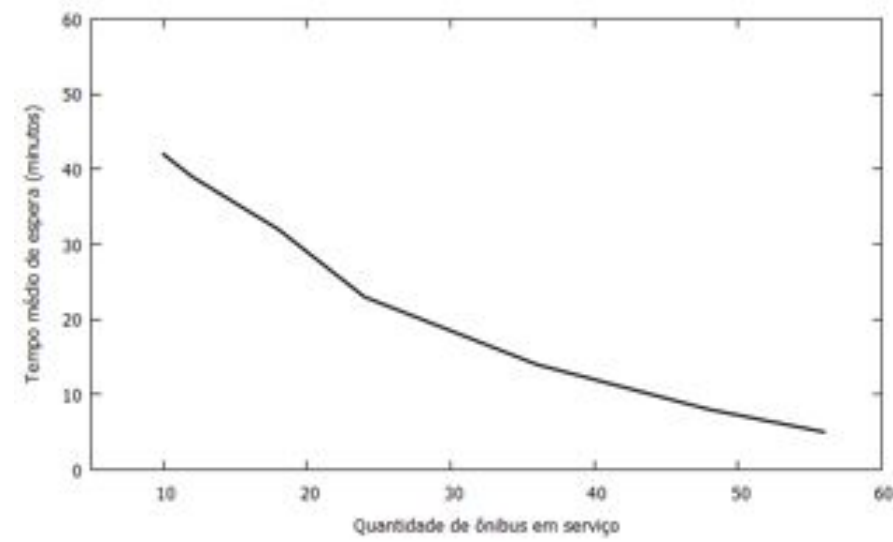

Figura 6. Tempo de espera de um usuário por um ônibus na jornada de 24 horas em fluxo ampliado

\section{Conclusões e perspectivas futuras}

Este artigo apresentou um trabalho em curso que descreve uma ferramenta de simulação para permitir o monitoramento do processo de transporte urbano. As funcionalidades implementadas pela ferramenta são a detecção de comboios e geração de relatório com informações estatísticas sobre os carros mais atrasados e as linhas com mais sobrecarga. A ferramenta é suportada pela concepção de agentes de software, que permite a atribuição de comportamentos individuais a cada dos seus elementos.

Uma vez concluída a implementação da ferramenta de simulação, a ideia é incorporar um comportamento diferente para os motoristas por agentes de software. Além disso, tais informações e informações detalhadas sobre o posicionamento de carros podem ser utilizadas para análise do comportamento do tráfego na modelagem de demanda futura. A ferramenta apresentada neste artigo pode ser usada com outras ferramentas para aplicação aos sistemas de transporte público com a finalidade de melhorar a $Q o S$ de trânsito e transporte de passageiros. Adicionalmente, para trabalhos futuros, a ideia é usar a expertise obtida neste trabalho para a construção de uma ferramenta para monitoramento em tempo real e trabalhar com o apoio de dados verídicos. 


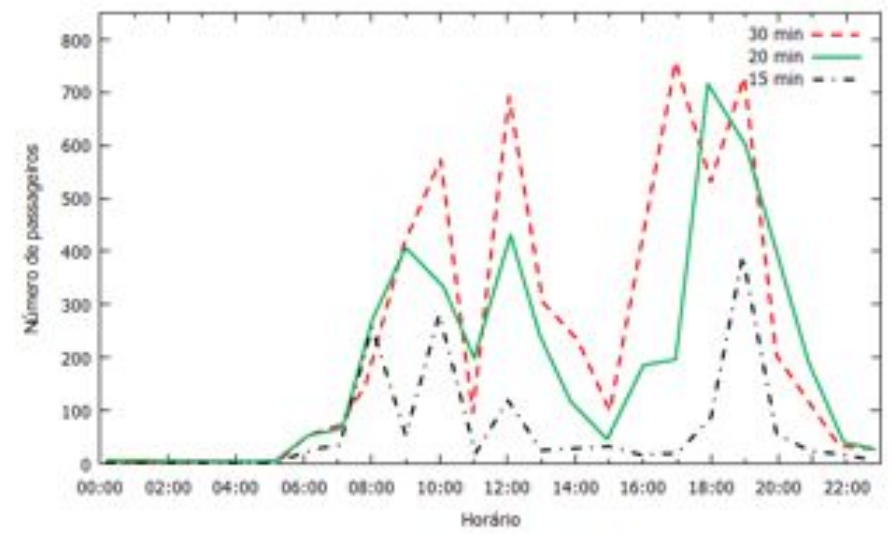

Figura 7. Quantidade de passageiros por ônibus na jornada de $\mathbf{2 4}$ horas em fluxo ampliado (média de todas as linhas)

\section{Agradecimentos}

Este trabalho está sendo conduzido no escopo dos projetos CTIC SIMTUR Cidades Inteligentes - RNP e CNPq/FAPERGS RS-SOC PRONEX número 10/0049-7.

\section{Referências}

Castro da Silva, B.; Bazzan, A. L. C.; Andriotti, G. K.; Lopes, F.; de Oliveira, D. (2004) "ITSUMO: an intelligent transportation system for urban mobility" in Proceedings of the 4th Int. Conf. on Innovative Internet Community Systems (IICS'04),pp. 224-235.

Behrisch, M.; Bieker, L., Erdmann, J.; Krajzewicz, D. (2011) "SUMO - Simulation of Urban MObility: An Overview" in SIMUL 2011, The Third International Conference on Advances in System Simulation.

Gruer, P., Hilaire, V., Koukam, A. (2001) "Multi-agent approach to modeling and simulation of urban transportation system", in IEEE Systems, Man, and Cybernetics Conference. pp. 2499-2504.

Ladeira, M. C. M.; Michel, F. D., Pavanatto, S. A. (2009) "Monitoramento da operação de transporte público: o caso de Porto Alegre", in Congresso de Pesquisa e Ensino em Transportes.

Martin, M.; Nurmi, P. (2006) "A generic large-scale simulator for ubiquitous computing", in Mobile and Ubiquitous Systems, Annual Int. Conference on, 0:1-3.

Meignan, D.; Simonin, O.; Koukam, A. (2007) "Simulation and evaluation of urban bus networks using a multi agent approach", in Simulation Modeling Practice and Theory V. 15, N. 6, pp. 659-671.

Molina, M. (2005) "An intelligent assistant for public transport management", in Proceedings of International Conference on Intelligent Computing, ICIC 2005, pp. 199-208.

Staib, R.; Gerland, H. (1999) "Advanced transit fleet management transfer of European systems and solutions into American environment", in Proceedings of the 1999 APTA Bus Conference. pp. 136-143. 